Supporting Information

\title{
Simultaneous preconcentration and desalting of organic solutes in aqueous solutions by bubble bursting
}

Konstantin Chingin*, Yunfeng Cai, Juchao Liang, and Huanwen Chen*

Jiangxi Key Laboratory for Mass Spectrometry and Instrumentation, East China University of Technology, Nanchang 330013, P. R. China

Corresponding authors: Dr. Konstantin Chingin and Dr. Huanwen Chen

E-mail: chingin.k@gmail.com; chw8868@gmail.com.

Room 804, Sci. \& Tech. Building

418 Guanglan Road, Nanchang City, Jiangxi Province 330013, P. R. China

Tel: (+86)791-8389-6370. Fax: (+86)791-8389-6370. 


\section{Experimental}

Bursting bubbles were generated at ambient conditions either by sonicating aqueous solutions $\left(10-500 \mathrm{~mL}\right.$ ) pre-saturated with $\mathrm{CO}_{2}$ gas (dry ice, $\sim 100 \mathrm{mg} / \mathrm{mL}$ ) or by feeding gas through the porous surface (i.d. $100 \mu \mathrm{m}$ ) at the bottom of the aqueous solution (Figure S-1). In the first approach the average bubble diameter and bubbling rate at $10 \mathrm{~cm}$ distance from the bottom were roughly $0.5 \mathrm{~mm}$ and ca. 50 bubbles per $1 \mathrm{~cm}^{2}$ per $1 \mathrm{~s}$ accordingly. In the second approach the average bubble diameter and bubbling rate at $10 \mathrm{~cm}$ distance from the bottom were roughly $0.7 \mathrm{~mm}$ and ca. 100 bubbles per $1 \mathrm{~cm}^{2}$ per $1 \mathrm{~s}$ accordingly. The bursting bubble aerosol was collected using a slanted glass slide fixed above the liquid surface. Typically, ca. $100 \mu \mathrm{L}$ of aerosol was collected within 5-10 min from $500 \mathrm{~mL}$ bulk solution. No bubble foam of multiple layers was formed at the solution surface interface during bubbling in the experiments reported in this study. However, for some surface active compounds, particularly proteins, foam was formed at high analyte concentration $(>100 \mu \mathrm{M})$. Foam formation greatly hinders bubble bursting efficiency and should be avoided by using appropriate sample dilution. The ratio of collected aerosol solution volume to the volume of the bulk solution removed by bubbling was ca. 9:10. The 10\% volume loss could possibly be related either to the collection efficiency or to the partial evaporation of water from the aerosol droplets.

Collected aerosols and bulk solutions were analyzed in three technical replicates under identical experimental conditions by direct-infusion ESI-MS (LTQ, Thermo Scientific, San Jose, CA) in positive ion detection mode using built-in loop injector $(20 \mu \mathrm{L})$, unless specified otherwise. Analyte R-values were derived as the intensity ratios of corresponding signals in the collected aerosol and bulk sample $\left(R=1^{\text {bubble }} / I^{\text {bulk }}\right)$. If necessary, solutions were accordingly diluted prior to MS analysis for the highest linearity of instrument response. For model single-analyte solutions, analyte quantification was performed using 
calibration response curve built using ESI-MS analysis of reference standard dilution samples (Figure S-8).

\section{Chemical partitioning during bubble bursting process}

As the bursting bubbles carry away pre-concentrated analytes, the number of analyte molecules in the bulk (N) as well as the bulk volume $(\mathrm{V})$ are both gradually decreasing. The relation between $\mathrm{N}$ and $\mathrm{V}$ can be expressed as:

$d N / d V=R^{*}(N / N)$

(eq. 1),

where $\mathrm{R}$ is the concentration enrichment factor for the specific analyte in bursting bubbles; $d \mathrm{~N}$ and $\mathrm{dV}$ are the differentials of $\mathrm{N}$ and $\mathrm{V}$ (both have negative magnitude).

Integration of eq. 1 yields the following explicit dependence of $\mathrm{N}$ on $\mathrm{V}$ :

$N=N_{0}\left(V / V_{0}\right)^{R}$

where $V_{0}$ is the initial bulk volume and $N_{0}$ is the initial number of analyte molecules in the bulk. From eq. 2 the analyte concentration can be derived:

$\mathrm{C}=\mathrm{N} / \mathrm{V}=\mathrm{C}_{0}\left(\mathrm{~V} / \mathrm{V}_{0}\right)^{\mathrm{R}-1}=\mathrm{C}_{0}\left(1-\Delta \mathrm{V} / \mathrm{V}_{0}\right)^{\mathrm{R}-1}$

Where $\Delta \mathrm{V}(\mathrm{t})=\mathrm{V}_{0}-\mathrm{V}(\mathrm{t})$ is the volume removed from the bulk solution by bursting bubbles by the time $t$. 


\section{Supplementary Tables}

Table S-1. Enrichment factor $\left(R=1^{\text {bubble } / I^{\text {bulk }}}\right)$ of analytes by bursting bubbles in single-analyte aqueous solutions. Bubbles were produced by ultrasound (Figure S-1a). Bubble path length was $20 \mathrm{~cm}$. Relative analyte quantification was performed using calibration response curve built using ESI-MS analysis of reference standard dilution samples (Figure S-8). Phe = phenylalanine; EtOAc = ethanol aceate; Ins = insulin; His = histidine; Mel = melamine; Qui = quinine; Mb = myoglobin; Gly = glycine; Lyz = lysozyme; Arg = arginine; Lys = lysine; Pyr = pyridine; DNA = CGATAGC

\begin{tabular}{|c|c|c|c|c|c|c|c|c|c|}
\hline & $\begin{array}{c}\text { Phe } \\
(100 \mu \mathrm{M})\end{array}$ & $\begin{array}{l}\text { EtOAc } \\
(50 \mu \mathrm{M})\end{array}$ & $\begin{array}{c}\text { Ins } \\
(1 \mu \mathrm{M})\end{array}$ & $\begin{array}{c}\text { His } \\
(100 \mu \mathrm{M})\end{array}$ & $\begin{array}{c}\text { Mel } \\
(100 \mu \mathrm{M})\end{array}$ & $\begin{array}{c}\text { Qui } \\
(50 \mu \mathrm{M})\end{array}$ & $\begin{array}{c}M b \\
(3 \mu \mathrm{M})\end{array}$ & $\begin{array}{c}\text { Gly } \\
(100 \mu \mathrm{M})\end{array}$ & $\begin{array}{c}\text { Lyz } \\
(1 \mu \mathrm{M})\end{array}$ \\
\hline $\mathbf{R}$ & $56 \pm 15$ & $52 \pm 10$ & $43 \pm 16$ & $37 \pm 9$ & $37 \pm 13$ & $34 \pm 12$ & $30 \pm 5$ & $28 \pm 5$ & $27 \pm 8$ \\
\hline & $\begin{array}{c}\text { Arg } \\
(30 \mu \mathrm{M})\end{array}$ & $\begin{array}{c}\text { Lys } \\
(100 \mu \mathrm{M})\end{array}$ & $\begin{array}{c}\text { Pyr } \\
(50 \mu \mathrm{M})\end{array}$ & $\begin{array}{r}\text { D } \\
\text { (CGATA }\end{array}$ & $\begin{array}{l}\text { A } \\
\text { C) }(1 \mu \mathrm{M})\end{array}$ & $\begin{array}{c}\text { Urea } \\
(200 \mu \mathrm{M})\end{array}$ & $\begin{array}{c}\text { CsI } \\
(10 \mu \mathrm{M})\end{array}$ & $\begin{array}{c}\mathrm{NaCl} \\
(100 \mu \mathrm{M})\end{array}$ & $\begin{array}{c}\mathrm{AgNO}_{3} \\
(300 \mu \mathrm{M})\end{array}$ \\
\hline $\mathbf{R}$ & $23 \pm 5$ & $20 \pm 6$ & $13 \pm 2$ & & \pm 2 & $15 \pm 4$ & $1.1 \pm 0.1$ & $1.0 \pm 0.1$ & $1.0 \pm 0.1$ \\
\hline
\end{tabular}


Table S-2. Signal enrichment $\left(R=1^{\text {bubble }} /\left.\right|^{\text {bulk }}\right)$ for the chemicals in a water mixture depending on the path length of rising bubbles. Bubbles were produced by gas pumping (Figure S-1b). Pyr = pyridine; Mel = melamine; Phe = phenylalanine; $\operatorname{Arg}=$ arginine; Qui = quinine .

\begin{tabular}{lcccccc}
\hline & $\begin{array}{c}\text { Pyr } \\
(\mathbf{5} \boldsymbol{\mu M})\end{array}$ & $\begin{array}{c}\text { Me1 } \\
\mathbf{( 3 0 0} \boldsymbol{\mu M})\end{array}$ & $\begin{array}{c}\text { CsI } \\
\mathbf{( 4 0 0} \boldsymbol{\mu M})\end{array}$ & $\begin{array}{c}\text { Phe } \\
(\mathbf{5 0 0} \boldsymbol{\mu M})\end{array}$ & $\begin{array}{c}\text { Arg } \\
(\mathbf{1 0 0} \boldsymbol{\mu M})\end{array}$ & $\begin{array}{c}\text { Qui } \\
(\mathbf{3 0 0} \boldsymbol{\mu M})\end{array}$ \\
\hline $\mathbf{R}_{\mathbf{1 7 0} \mathbf{c m}}$ & $4 \pm 1$ & $5 \pm 1$ & $0.8 \pm 0.1$ & $6 \pm 1$ & $5 \pm 1$ & $15 \pm 4$ \\
$\mathbf{R}_{\mathbf{1 3 0} \mathbf{c m}}$ & $9 \pm 2$ & $5 \pm 1$ & $0.82 \pm 0.1$ & $7 \pm 2$ & $6 \pm 1$ & $58 \pm 16$ \\
$\mathbf{R}_{\mathbf{9 0} \mathbf{c m}}$ & $15 \pm 3$ & $9 \pm 2$ & $0.77 \pm 0.1$ & $14 \pm 3$ & $12 \pm 2$ & $101 \pm 20$ \\
$\mathbf{R}_{\mathbf{3 0}} \mathbf{c m}$ & $20 \pm 4$ & $22 \pm 4$ & $0.9 \pm 0.1$ & $27 \pm 4$ & $17 \pm 3$ & $28 \pm 8$ \\
$\mathbf{R}_{\mathbf{1 0} \mathbf{c m}}$ & $10 \pm 2$ & $13 \pm 3$ & $1 \pm 0.1$ & $16 \pm 3$ & $10 \pm 2$ & $16 \pm 5$ \\
\hline
\end{tabular}

Table S-3. Signal enrichment $\left(R=1^{\text {bubble }} /\left.\right|^{\text {bulk }}\right)$ for the chemicals in water mixture depending on the gas used to induce bubbling. Bubbles were produced by the setup shown in Figure S-1b. Bubble path length was $90 \mathrm{~cm}$. Pyr = pyridine; Mel = melamine; Phe = phenylalanine; $\operatorname{Arg}=$ arginine; Qui $=$ quinine.

\begin{tabular}{|c|c|c|c|c|c|c|}
\hline & $\begin{array}{c}\text { Pyr } \\
(5 \mu \mathrm{M})\end{array}$ & $\begin{array}{c}\text { Mel } \\
(300 \mu \mathrm{M})\end{array}$ & $\begin{array}{c}\text { Csl } \\
(400 \mu \mathrm{M})\end{array}$ & $\begin{array}{c}\text { Phe } \\
(500 \mu \mathrm{M})\end{array}$ & $\begin{array}{c}\text { Arg } \\
(100 \mu \mathrm{M})\end{array}$ & $\begin{array}{c}\text { Qui } \\
(300 \mu \mathrm{M})\end{array}$ \\
\hline $\mathbf{R}\left(\mathbf{N}_{2}\right)$ & $15 \pm 3$ & $9 \pm 3$ & $0.77 \pm 0.1$ & $14 \pm 3$ & $12 \pm 3$ & $101 \pm 20$ \\
\hline $\mathrm{R}\left(\mathrm{CH}_{2}=\mathrm{CH}_{2}\right)$ & $11 \pm 2$ & $7 \pm 2$ & $0.88 \pm 0.1$ & $11 \pm 2$ & $8 \pm 2$ & $81 \pm 12$ \\
\hline $\mathrm{R}\left(\mathrm{CO}_{2}\right)$ & $11 \pm 2$ & $33 \pm 5$ & $0.92 \pm 0.1$ & $12 \pm 3$ & $10 \pm 3$ & $35 \pm 9$ \\
\hline
\end{tabular}


Table S-4. Analytes identified in a mosquito spray by GC-MS (Figure 3).

\begin{tabular}{|c|c|c|c|c|}
\hline No. & Analyte & $\begin{array}{l}\text { Molecular } \\
\text { Structure }\end{array}$ & $\begin{array}{l}\text { Retention } \\
\text { time (min) }\end{array}$ & $\begin{array}{c}\text { Major } \\
\text { fragments } \\
\text { in MS } \\
(\mathbf{m} / \mathbf{z})\end{array}$ \\
\hline 1 & $\beta$-pinene & & $5.00-5.06$ & $\begin{array}{c}136,121, \\
93,79,69, \\
41\end{array}$ \\
\hline 2 & hexyl formate & & $5.78-5.84$ & $\begin{array}{l}84,73,69, \\
61,56,43\end{array}$ \\
\hline 3 & dipentene & & $6.34-6.40$ & $\begin{array}{c}136,121, \\
107,93, \\
79,68,53, \\
41\end{array}$ \\
\hline 4 & linalool & & $8.41-8.48$ & $\begin{array}{c}136,121, \\
93,80,71, \\
55,43\end{array}$ \\
\hline 5 & benzyl acetate & & $10.40-10.50$ & $\begin{array}{r}150,108, \\
91,79,65, \\
51,43,39\end{array}$ \\
\hline 6 & menthol & & $10.70-10.85$ & $\begin{array}{c}138,123, \\
109,95, \\
81,71,55, \\
43,41\end{array}$ \\
\hline 7 & 4-methylbenzyl acetate & & $11.36-11.41$ & $\begin{array}{c}164,122, \\
104,77 \\
51,43,39\end{array}$ \\
\hline 8 & 4-tert-butylcyclohexyl acetate & & $14.31-14.70$ & $\begin{array}{c}138,123, \\
82,67,61, \\
57,43\end{array}$ \\
\hline 9 & $\gamma$-decalactone & & $19.00-19.03$ & $\begin{array}{r}128,100, \\
85,55,41\end{array}$ \\
\hline 10 & $\begin{array}{c}\text { oxalic acid, } \\
\text { di(1-menthyl)ester }\end{array}$ & & $19.36-19.53$ & $\begin{array}{c}139,123, \\
97,83,81 \text {, } \\
69,55,41\end{array}$ \\
\hline
\end{tabular}




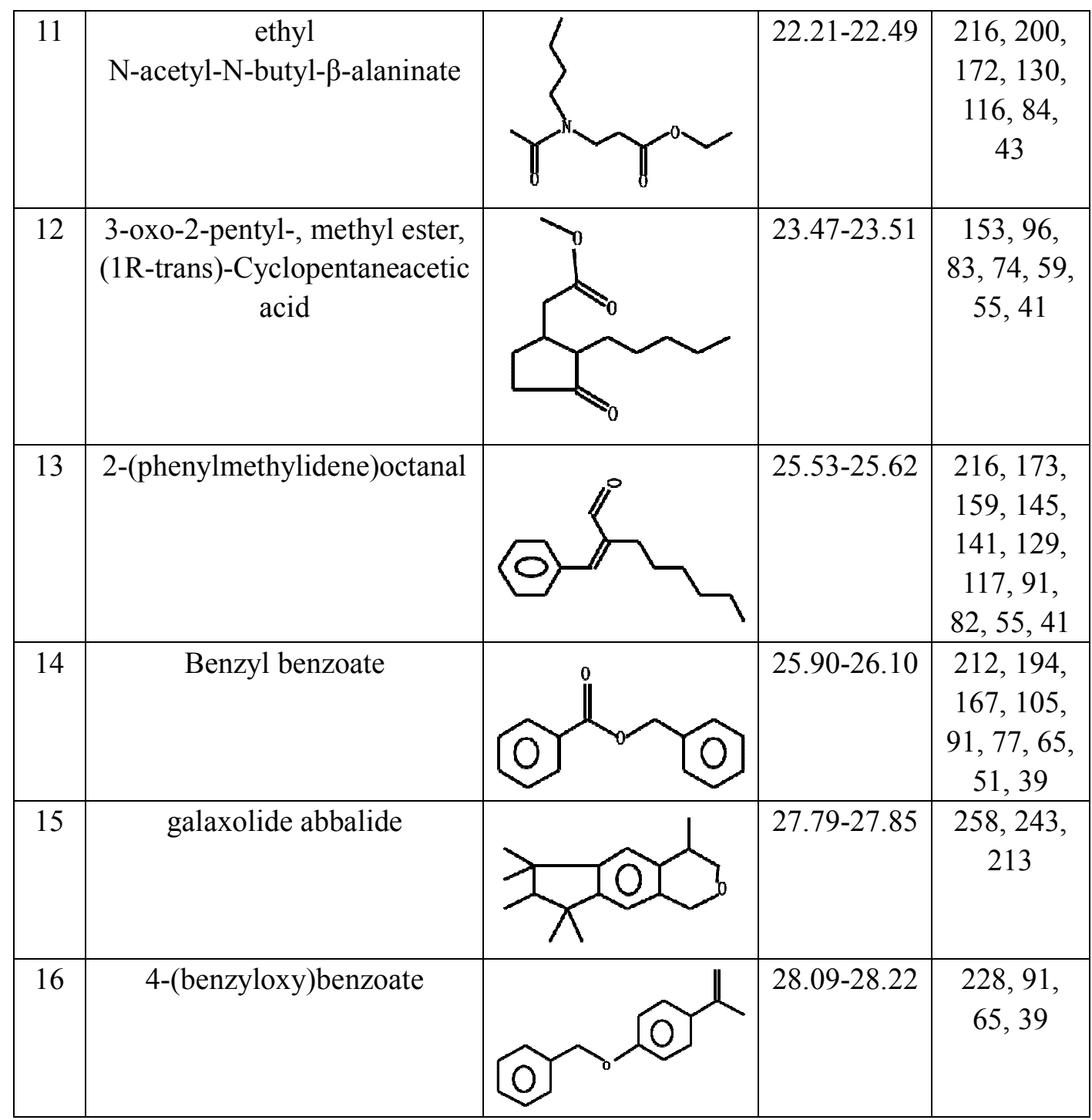




\section{Supplementary Figures}

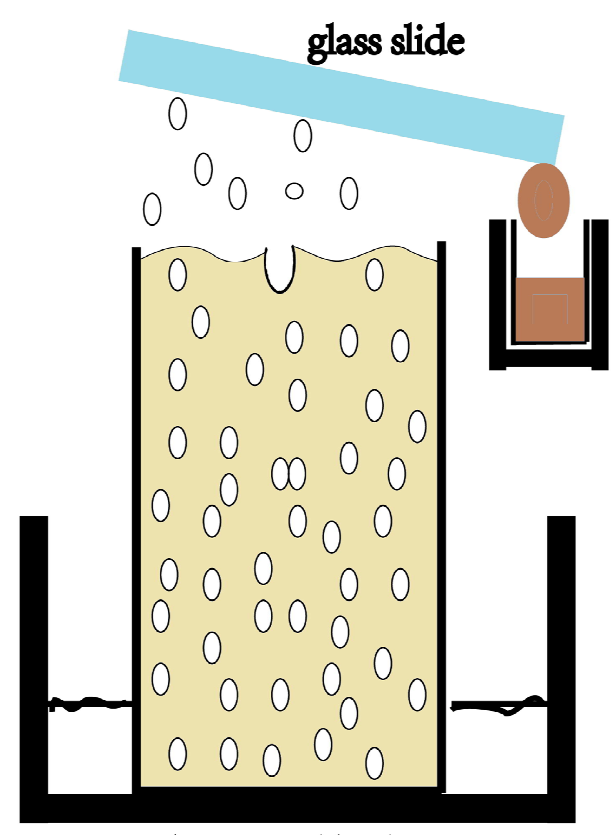

ultrasound bath

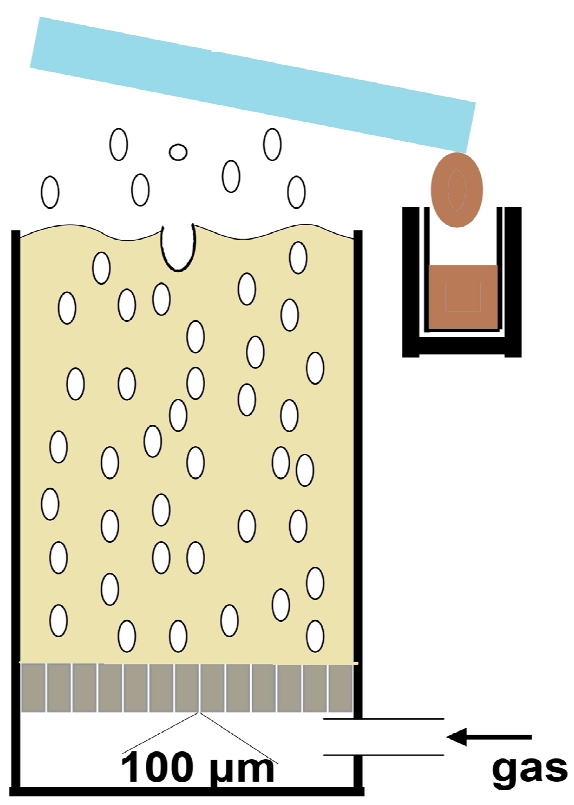

(b)

Figure S-1. Generation of bursting bubbles at ambient conditions: a) by sonicating aqueous analyte solutions pre-saturated with $\mathrm{CO}_{2}$ gas; b) by feeding gas through the porous surface at the bottom of the aqueous analyte solution. 


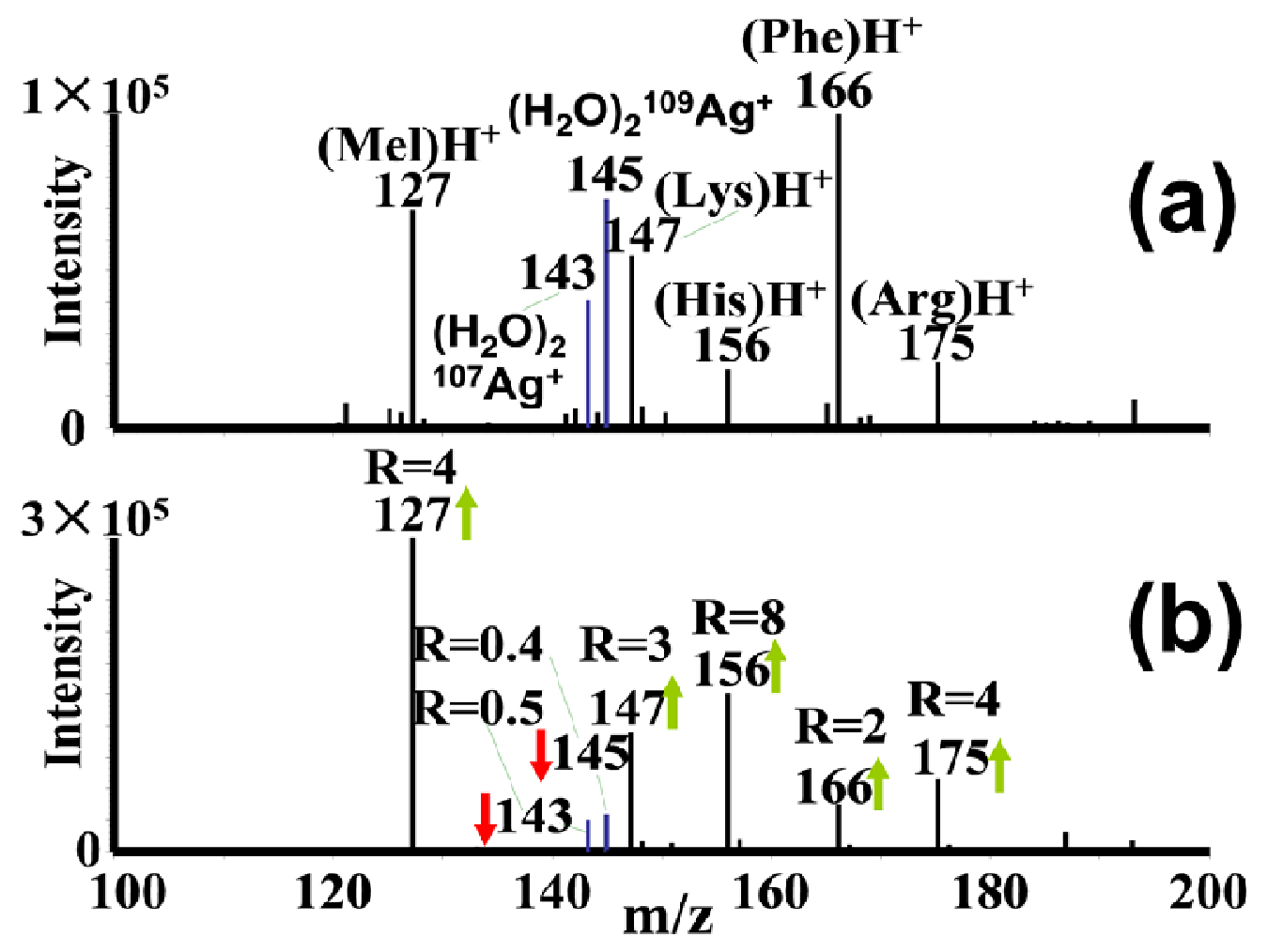

Figure S-2. a) Direct-infusion ESI-MS analysis of a model analyte solution in water $(500 \mathrm{~mL})$ containing arginine (Arg, $80 \mu \mathrm{M})$, histidine (His, $50 \mu \mathrm{M})$, lysine (Lys, $100 \mu \mathrm{M}$ ), phenylalanine (Phe, $500 \mu \mathrm{M}$ ), melamine (Mel, $300 \mu \mathrm{M}$ ), silver nitrate (AgNO3, $500 \mu \mathrm{M})$; b) direct-infusion ESI-MS of a bubble aerosol (100 $\mu \mathrm{L}$ ) collected from the same original solution over $5 \mathrm{~min}$ and analyzed under identical MS parameters. Bubbles were produced by ultrasound (Figure S-1a). Bubble path length was $20 \mathrm{~cm}$. 


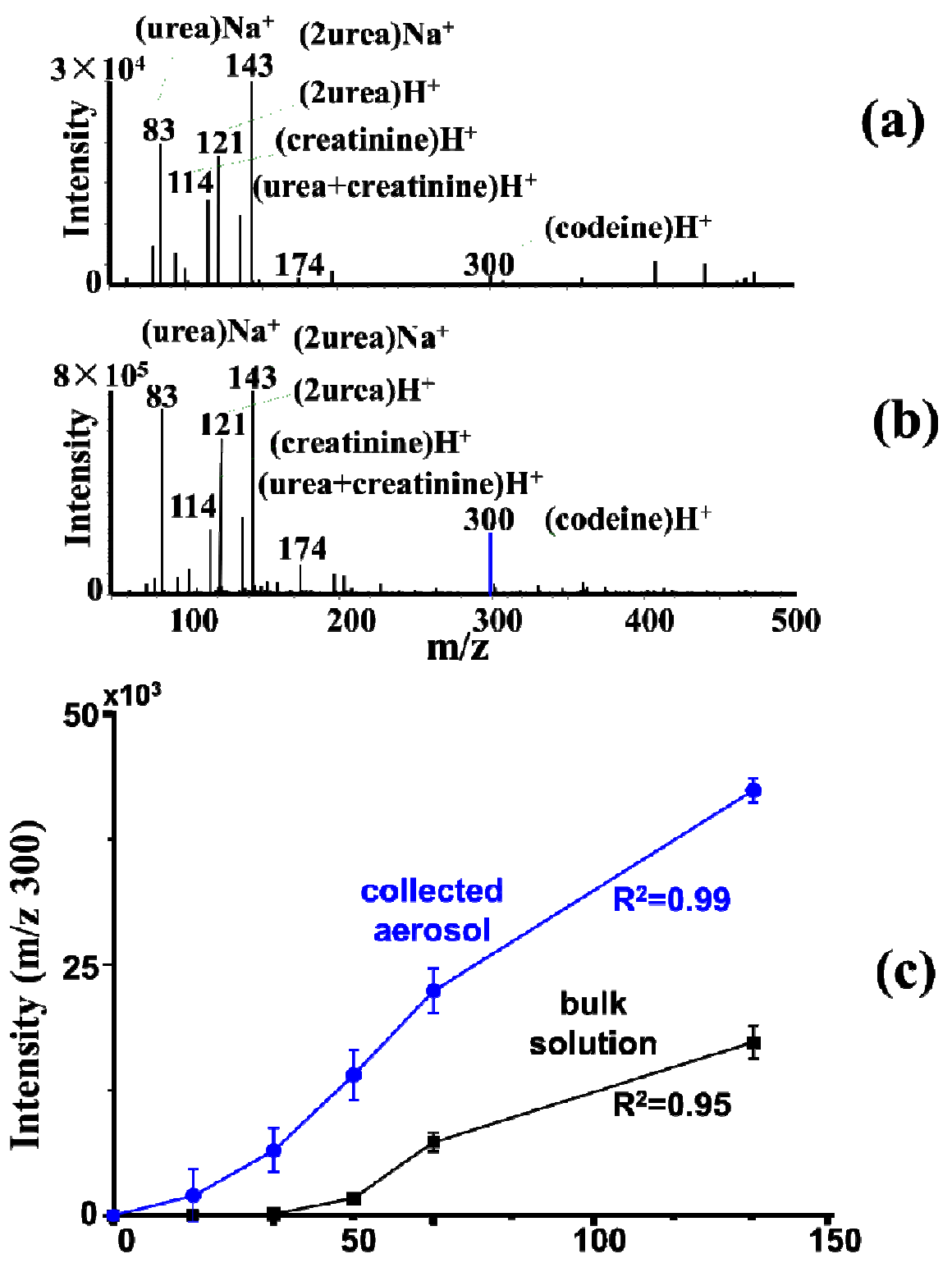

Figure S-3: a) Direct-infusion ESI-MS of urine spiked with $20 \mu \mathrm{M}$ codeine and diluted 1000 times in water; b) direct-infusion ESI-MS of a bubble aerosol collected from the same original solution; $c$ ) concentration response curve for the codeine signal $(\mathrm{m} / \mathrm{z} 300)$. Bubbles were produced by ultrasound (Figure $\mathrm{S}-1 \mathrm{a})$. Bubble path length was $20 \mathrm{~cm}$. 


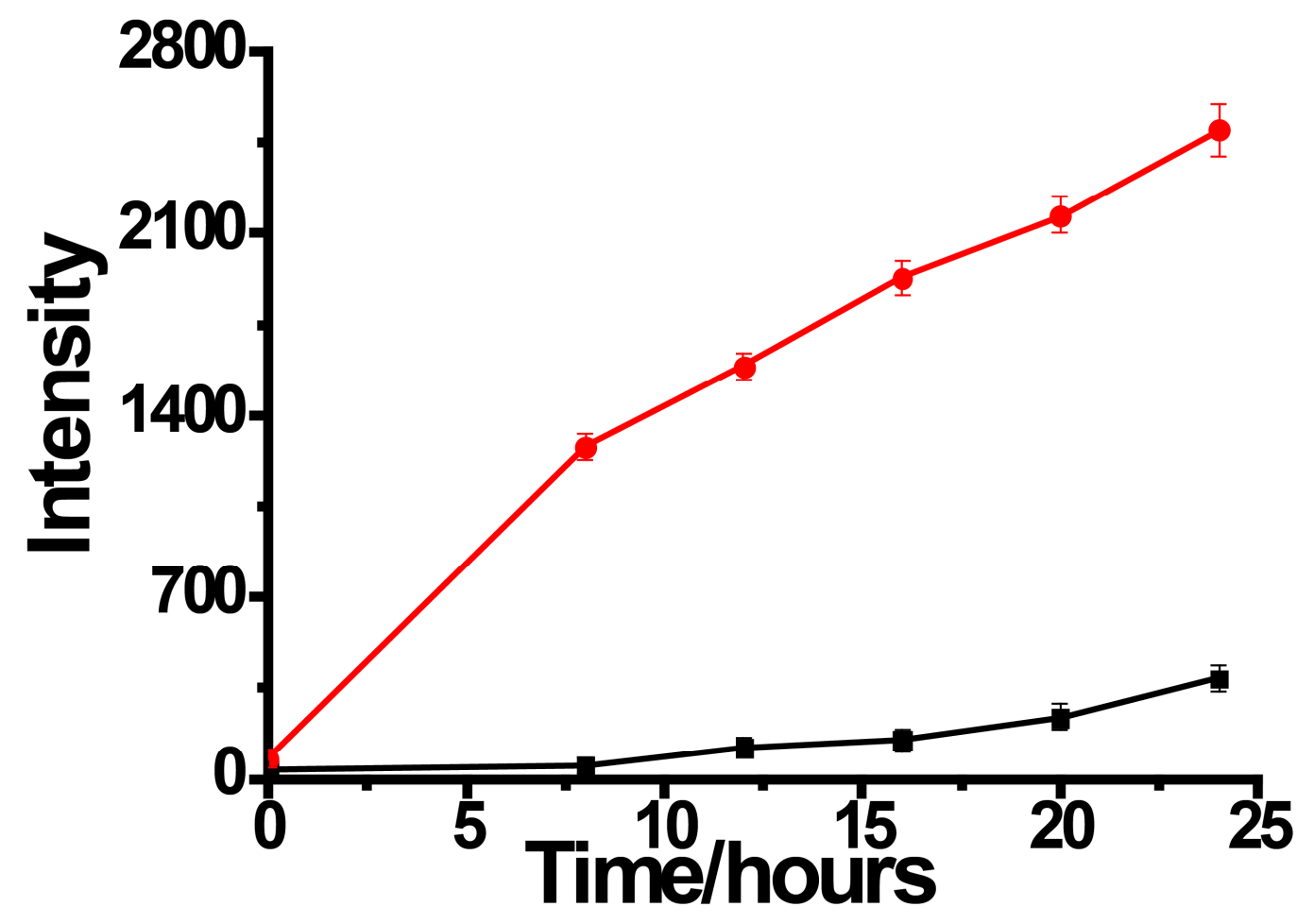

Figure S-4: The time profile of signal intensity for isovaleric acid $(\mathrm{m} / \mathrm{z} 101)$ measured in Staphylococcus aureus bacterial culture in water (black curve) and in the collected aerosol bacterial culture (red). Bubbles were produced by ultrasound (Figure S-1a). Bubble path length was $20 \mathrm{~cm}$. All the samples were analyzed by direct-infusion ESI-MS and diluted 100 times prior to analysis. 


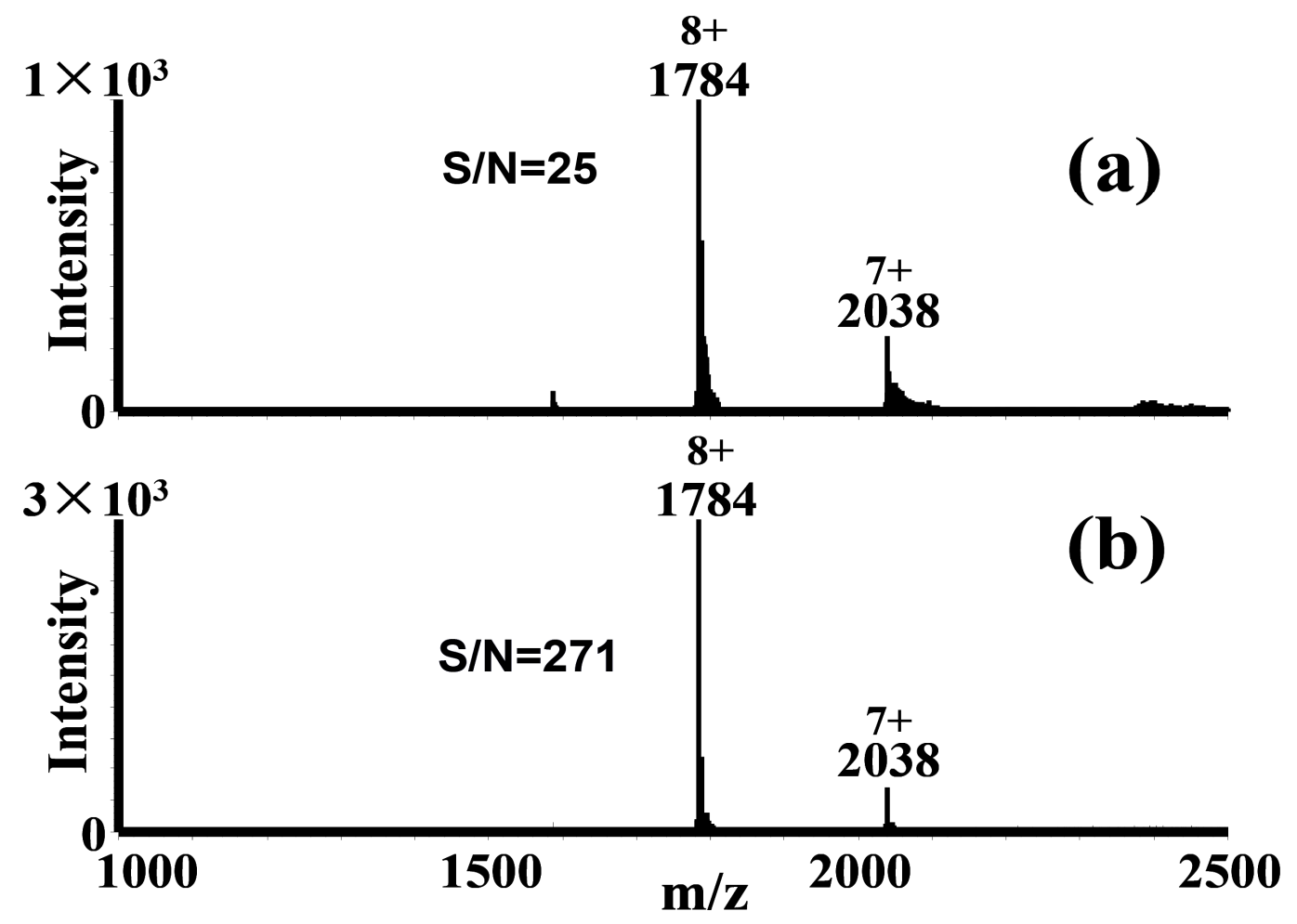

Figure S-5: a) Direct-infusion ESI-MS of a model analyte solution containing 1 $\mu \mathrm{M}$ lysozyme and $1 \mathrm{mM} \mathrm{NaCl}$ in $20 \mathrm{mM}$ aqueous $\mathrm{NH}_{4} \mathrm{Ac}$; b) direct-infusion ESI-MS of a bubble aerosol collected from the same original solution and diluted 10 times. Bubbles were produced by ultrasound (Figure S-1a). Bubble path length was $20 \mathrm{~cm}$. 


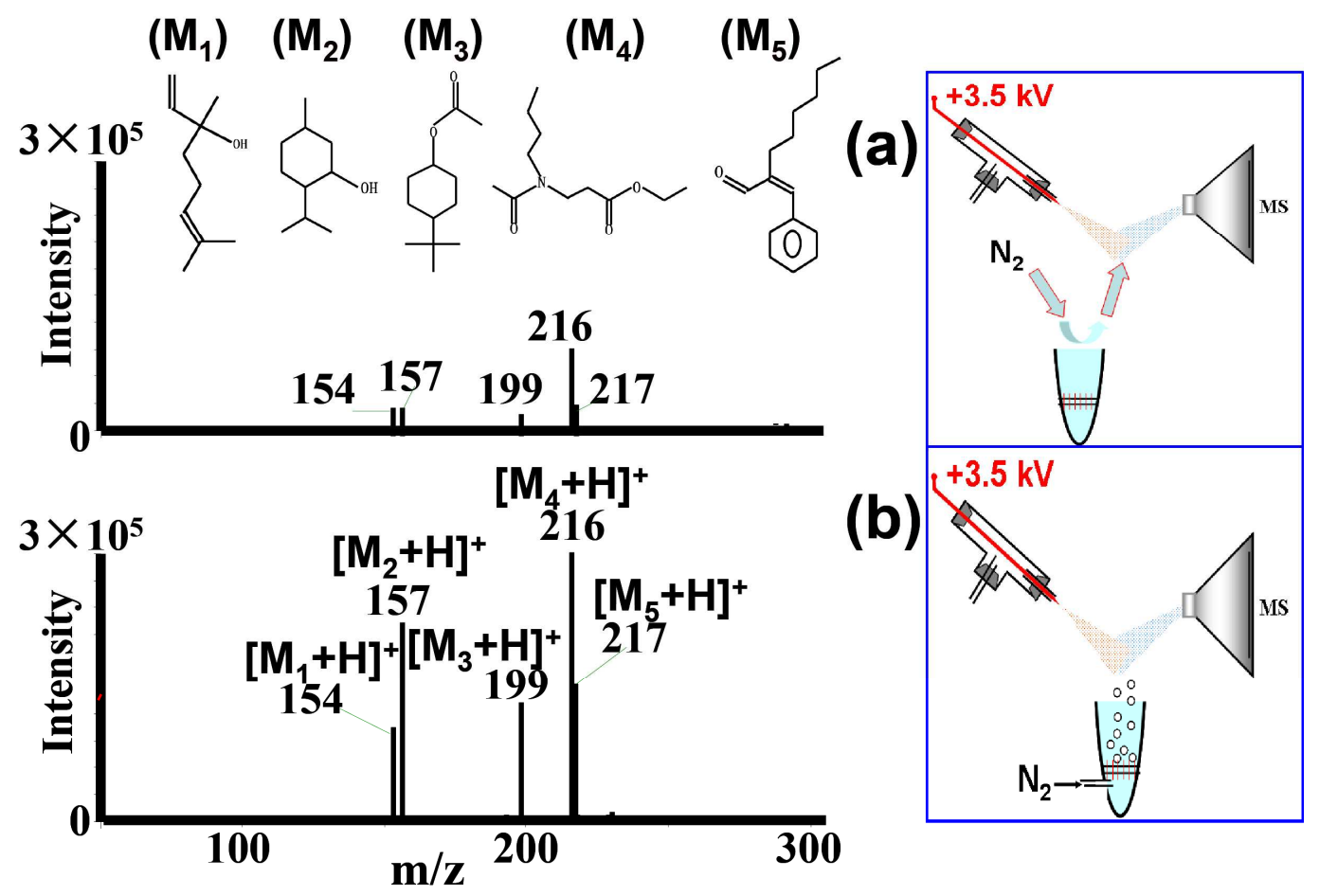

Figure S-6.EESI-MS of a mosquito spray: a) volatile fraction analysis; b) bursting bubble analysis. Bubbles were produced by the setup shown in Figure $\mathrm{S}-1 \mathrm{~b}$. Bubble path length was $5 \mathrm{~cm}$. Solution volume was $4 \mathrm{~mL}$. 

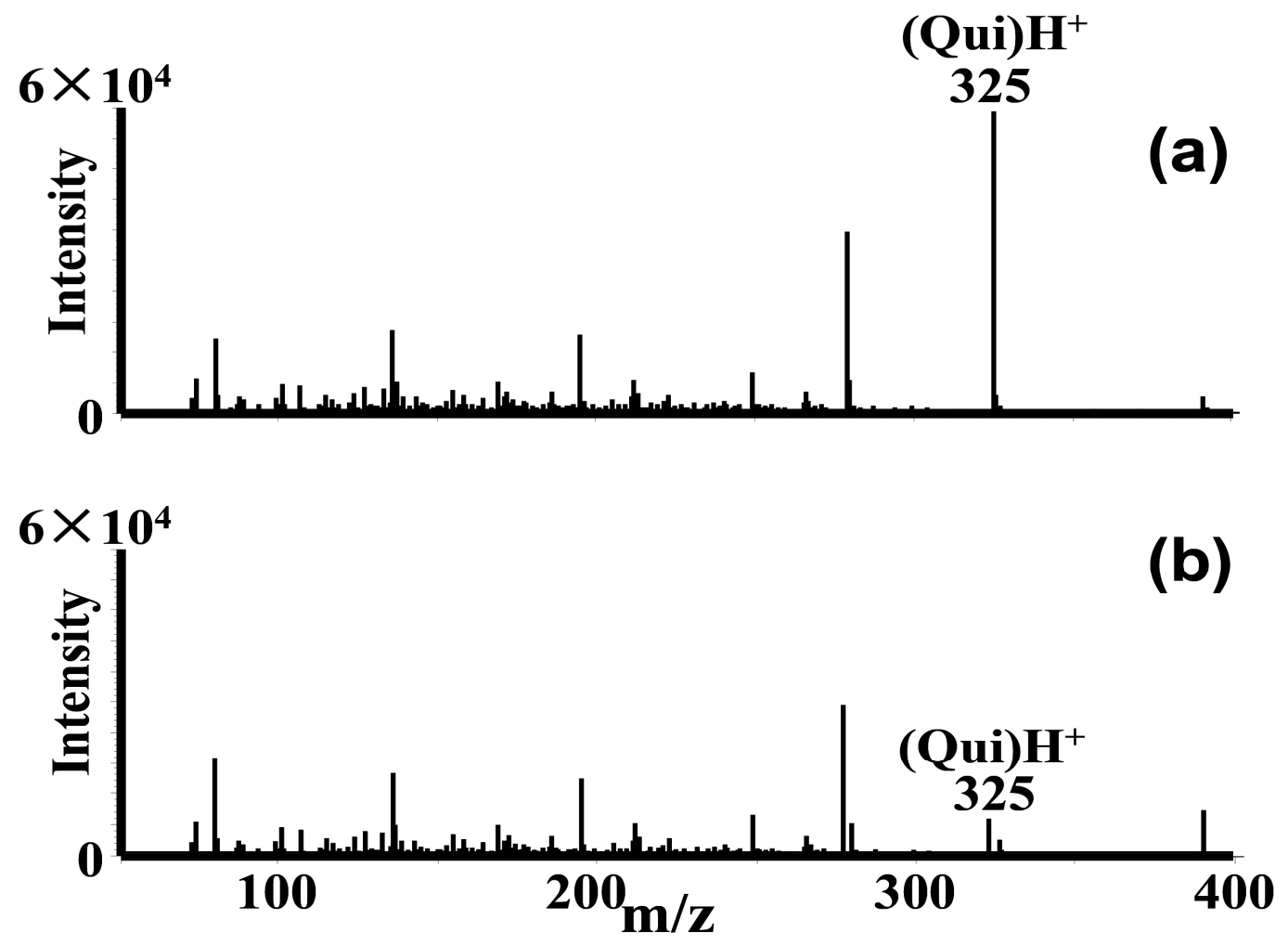

Figure S-7. Direct-infusion ESI-MS of a model aqueous solution containing 1 $\mu \mathrm{M}$ quinine $(\mathrm{R} \approx 95)$ before $(\mathrm{a})$ and after $(\mathrm{b})$ the removal of $2 \%$ volume by bubble bursting $\left(V_{0}=400 \mathrm{~mL} ; \Delta V=8 \mathrm{~mL}\right)$. Ca. 7-times decrease in quinine signal intensity is observed. Bubbles were produced by gas pumping using the setup shown in Figure S-1b. Bubble path length was $90 \mathrm{~cm}$. 


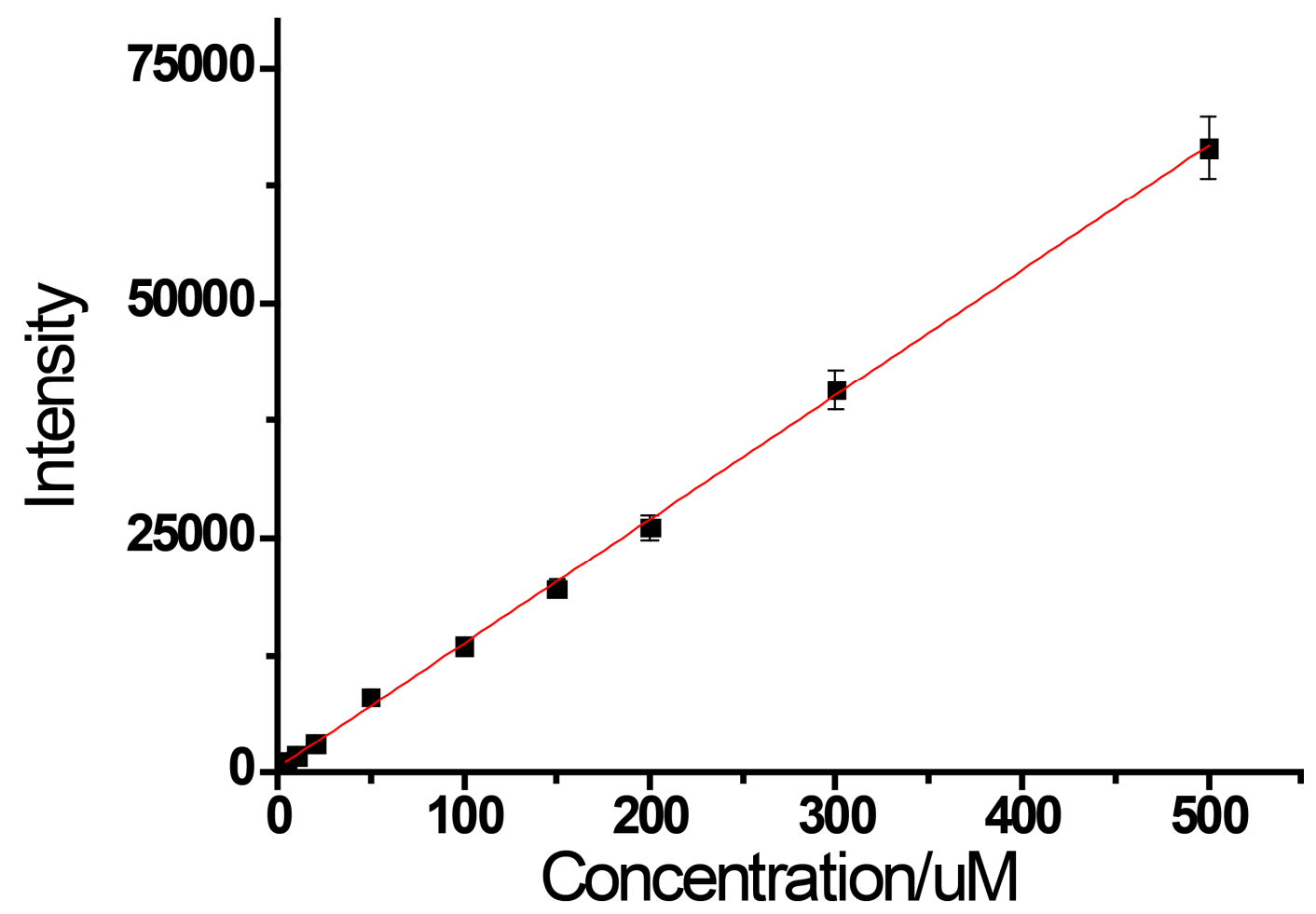

Figure S-8. Typical analyte concentration response curve built using ESI-MS analysis of single-analyte solutions in water with different analyte concentration. The linear range is shown for glucose analysis. Bubbles were produced by ultrasound (Figure S-1a). Bubble path length was $20 \mathrm{~cm}$. 